\title{
Synthesis of Modern Nanodrugs to Simulate the Activity of Cytochrome P 450 Enzymes Its Software Study to Enter the Surface Membrane of Blood
}

\section{sara Masoumi}

Islamic Azad University North Tehran Branch

Akbar Esmaeili ( $\nabla$ akbaresmaeili@yahoo.com)

Islamic Azad University North Tehran Branch

\section{Research Article}

Keywords: VMD and COMSOL software, magnetic nanoparticles, buprenorphine, releases, drug delivery

Posted Date: January 25th, 2022

DOI: https://doi.org/10.21203/rs.3.rs-1214695/v1

License: (c) (i) This work is licensed under a Creative Commons Attribution 4.0 International License.

Read Full License 


\section{Abstract}

In this study magnetic nanoparticles serve as a carrier for delivering the drug to the target. Simulations were done using COMSOL multi-physics and visual molecular dynamics software. In this study, with the help of docking, the structure of buprenorphine with CYP2C8 and CYP3A4 complexes was modeled to function as the main enzymes entering the human body (only model theoretical). The crystallographic structure of the complexes is available in the protein database. Interaction of BUP with cell membrane has been investigated in terms of Van der Waals energy and center of mass distance. Our results confirm the stability, appropriateness and low uptake of buprenorphine into the cell membrane. Computational results also confirmed the accuracy of laboratory studies. Due to its hydrophobic properties, buprenorphine retains stability and low adsorption by the cell membranes. Consumption and administration of buprenorphine can be much more effective and efficient in the treatment of withdrawal from modern drug delivery methods. The innovative feature of this project is the study of interactions using molecular dynamics simulation methods and the behavior of these complexes in different environments. Physiological simulations of the body for magnetic force and tensile force due to adhesion were examined utilizing visual molecular dynamics and COMSOL software.

\section{Introduction}

For a few decades now, one of the biggest problems for young people is dealing with addictive substances, which after a while, the body is able to quit and detoxify but only with great difficulty. In some cases, the body will be 'imprisoned' by a person's addiction for many years. According to some studies, buprenorphine (BUP) is one of the most important drugs in the field of addiction that is approved by the Food and Drug Administration authorities in the United States. BUP has been designated as the reference drug for our calculations ${ }^{1}$. The researchers examined the similarities and differences between BUP and norBUP which have unique BUP drug specifications. Kelly et al. ${ }^{2}$ studied the properties of metal optical nanoparticles in terms of the effect of size, shape and dielectric environment. It has approximately $96 \%$ protein binding and is metabolized by CYP3A4 and CYP2C8. BUP is marketed under the brand name Subutex. It is an opioid analgesic used to treat opioid disorders. Masoumi and Esmaeili ${ }^{3}$ explained the properties of cytochrome P450 enzymes in a new nanocarrier that incorporates BUP. A large proportion of P450 enzymes are responsible for catalyzing the oxidation process of organic compounds. Hatorp et al. 4 explained that the enzyme CYP plays a major role in the metabolism of drugs. Niemi et al. ${ }^{5}$ explained clarithromycin cytochrome P4503A4 inhibitor increases in concentration. In this study, using specific software calculations, the position and identification of the main purpose of this drug with the desired enzyme was investigated. This was done to show the best position and best connection to determine the appropriate complex to detoxify and help patients. The software used in this research is VMD and COMSOL software. Scheme 1 illustrates how COMSOL software can produce high quality videos and figures of different molecules in various types of cartoons, dots, surfaces, sticks and lines. Zimmerman ${ }^{6}$ asserted that COMSOL software is currently one of the most widely used macromolecular visualization tools. COMSOL software drug discovery function has been successfully applied to find new drug 
candidates for various purposes. In this paper, the focus of the software is simulated, the movements of drug-carrying nanoparticles in the vessel, and due to the proximity of blood and water density, it is selected to simplify the liquid-water environment. VMD and COMSOL software serve as a platform for designing computational drugs, and identify the appropriate binding site. In order to prevent unwanted side effects, the drug was deliberately transferred to healthy cells within the desired location. Among these factors the strength of the magnetic field of the external magnetic field was examined. In the meantime, accurate knowledge of this interaction can help in the treatment of behavioral and nonbehavioral diseases and disorders after quitting drugs.

\section{Experimental Section \\ 2.1. Synthesis of $\mathrm{Fe}_{3} \mathrm{O}_{4} \mathrm{NPS}$}

$1.99 \mathrm{~g}$ of $\mathrm{FeCl}_{2} \cdot 4 \mathrm{H}_{2} \mathrm{O}$ and $5.41 \mathrm{~g}$ of $\mathrm{FeCl}_{3} \cdot 6 \mathrm{H}_{2} \mathrm{O}$ with a molar ratio of $1: 2$ were dissolved in $100 \mathrm{ml}$ of water in the presence of nitrogen. The resulting solution was stirred at room temperature for $30 \mathrm{~min}$. Sodium oleate surfactant was added to the solution. When the solution temperature reached $500^{\circ} \mathrm{C}$, fresh ammonium hydroxide solution $(100 \mathrm{ml}, 6.4 \mathrm{M})$ was added dropwise to the solution. During the process of adding ammonia, the solution gradually changed color from orange to brown and then to charcoal, indicating the formation of nanoparticles $\left(\mathrm{Fe}_{3} \mathrm{O}_{4}\right)$. After the $\mathrm{pH}$ of the solution reached 11 , the addition of ammonia ceased. The solution was stirred for $30 \mathrm{~min}$ and subjected to $\mathrm{N}_{2}$ gas. Magnetite NPs were washed by repeated centrifugation and re-dispersion cycling in deionized water and ethanol. The nanoparticles were dried for $24 \mathrm{~h}$ at $40^{\circ} \mathrm{C}$ in an oven.

\subsection{Preparation of BUP with ascorbic acid with $\mathrm{Fe}_{3} \mathrm{O}_{4} \mathrm{NPs}$}

A load $0.5 \mathrm{~g}$ of NPs was added to $4 \mathrm{ml}$ of ethanol and then placed inan ultrasonic bath for $30 \mathrm{~min}$ and stirred for $24 \mathrm{~h} .0 .5 \mathrm{~g}$ of the NPs was mixed with $0.20 \mathrm{~g}$ of AA in $25 \mathrm{ml}$ of buffer solution $\mathrm{pH}=6.1(6.4 \mathrm{ml}$ of $0.1 \mathrm{M}$ sodium hydroxide solution and $50 \mathrm{ml}$ of $0.1 \mathrm{M}$ potassium phosphate dihydrogen solution). BUP is unstable in an acidic or alkaline environment and decomposes. The glass stirrer was subjected to 500 rpm at $37^{\circ} \mathrm{C}$. After $3 \mathrm{~h}$, the solution was then allowed to cool. It was then rinsed with distilled water and allowed to dry ${ }^{7}$.

\subsection{Coating by BUP modified NPs with natural polymer}

$5 \mathrm{~g}$ of the drug-carrying NPs was stirred in $10 \mathrm{ml}$ of $\mathrm{NaCl}$ to obtain a one-handed solution. The addition of $\mathrm{NaCl}$ was intended to increase the ionic strength of the solution. $20 \mathrm{ml}$ of ion sodium alginate solution (1 $\mathrm{g}$ of alginate was mixed in $99 \mathrm{ml}$ of water and $1 \mathrm{ml}$ of propanol for $2 \mathrm{~h}$ ) was added to the subsequent solution. This solution was stirred vigorously at $37^{\circ} \mathrm{C}$ for $1 \mathrm{~h}$ and then the solution was then washed thoroughly 8 .

\subsection{Identification and computational methods}




\subsubsection{Investigation and study of molecular docking method}

This research was performed computationally and analytically. MDM operations in order to predict the new composition and accurately identify the connection mechanism of the MDM using new data analysis software and 3D and 2D composition structures, were done to get the results. Its content was analyzed ${ }^{9}$. In this study, BUP was identified as the reference compound. VMD software was used for the MDM operations. 3D and crystalline structure of the enzyme along with the identification code CYP3A4 (CYP2C8) was selected from the database of references. Then in Zinc 15 database (ZINC100373348) the structure of the desired ligand and protein was identified and plotted ${ }^{10,11}$.

\subsubsection{Editing and macromolecular visualization and analysis of protein-ligand interactions (PLI)}

Together with VMD software, it is possible to edit a macromolecule, including changing the angles of the two sides, thus making a peptide. In VMD software a molecule was easily created from scratch. Real-time minimization of energy by VMD is another useful case in this research ${ }^{12}$. In this project, PLI interactions are essential for almost all biological processes in living organisms. Ligand-mediated signal transmission through molecular complement is essential for all life processes. Around a macromolecule to facilitate presentation, the coloring of the residues due to their hydrophilicity creates a quasi-atom at the center of the mass of selected atoms ${ }^{13}$. Next to PLI the main purpose of this modeling is to examine the fundamental interactions between ligands and specific receptors.

This is an important step in the discovery of modern drugs. PLI modeling includes protein homology modeling ligand preparation protein preparation, and PLI binding ${ }^{14}$. Crystal structures and homogeneous models have defects when preparing protein. To obtain reliable and accurate computational results, preparation of $\mathrm{PLI}$ is a mandatory step before any other MDM work that prepares the PLI in VMD. Following ligand preparation in PLI-based modeling, all ligands were optimized before doing other modeling tasks. Then we connect both of them. The purpose of PLI is to predict the position and orientation of the ligand and bind to a specific receptor. PLI binding has been extensively investigated and compared in optimization and guidance studies that looked at modern drug delivery ${ }^{15}$. Finally, in molecular simulation, the physical motions of atoms and molecules were analyzed.

\subsection{Method of simulating the movements and magnetic field of nanoparticles carrying drugs with COMSOL methods}

In this research, the movements of NPs with magnetic properties in a magnetic field were simulated using COMSOL software. Then an external magnetic field was defined and then the vessel was considered cylindrical with a certain length and cross-section. The next step was to start defining a system utilizing droplet delivery. The density close to water to the blood of the substance inside the vessel was chosen as water and the surrounding air was chosen. By moving the drop along the membrane, the drug dissolved in water. The final concentration was obtained by passing through the desired membrane and then by 
changing the droplet velocity. In the initial stage, the location of the membrane was deemed to be $Z=0.6$ $\mathrm{mm}$ and $\mathrm{Z}=0.8 \mathrm{~mm}$. The next step was to determine the empty spaces where air existed with a density of $1.25 \mathrm{~kg} \mathrm{~m}^{-3}$. The angle of contact of the drop with the wall was $135^{\circ}$, but in the permeable membrane section it amounted to $157.5^{0}$.

Then to consider was the inlet flux of the drug within the droplet as $1 \times 10^{-3} \mathrm{~mol} \mathrm{~m}^{2} \mathrm{~s}^{-1}$ ) and the coefficient of penetration of the drug into the water led to changes in droplet velocity from $0.1-1 \mathrm{~mm} \mathrm{~s}^{-1}$. The vessel stage was $16 \mathrm{~mm}$ long and $2 \mathrm{~mm}$ in diameter ${ }^{16,17}$. The drug particles penetrated through the membrane into the droplet because of the speed at which the fluid was injected. The velocity of the blade was fast until our convection occurred according to the velocity flow. Then, the penetration coefficient gave a value and the determined value according to the desired drug and the characteristics of its structure ${ }^{18}$. The flow line originated from the desired velocity. The velocity flow lines emerged from the point of entry of the drop and the point of exit of the drop in Figure 1.

\section{Results And Discussion}

\subsection{Investigation of structure and function of identified proteins}

Cytochrome P450 3A4 bound to an inhibitor with 4 D7D/1PQ2 crystal structure of human (only model theoretical) drug metabolizing cytochrome P450 2C8 in interaction with BUP receptor, is a minor agonist in opioid receptor and antagonist in kappa-opioid receptor. Samuels et al. ${ }^{19}$ made comparisons in their work to confirm the structure. Aquaporin is a group of membrane proteins and it acts as water channel and usually transports water at a speed comparable to the free infiltration of water. The target in this study depends on the structure. It shows the two sides of the protein. $Q=1$ is a perfect match to the molecular structure. When $\mathrm{Q}$ indicates a small value, then it is evident that the structures do not fit well together. Figure 1 shows that if there was no match, then more red colors would be seen. Some parts of

the concave lines in the figure are also red. Denker et al. ${ }^{20}$ examined the VMD molecules which demonstrated more or less similarities. The outcome of comparing two different types of proteins by VMD revealed that the molecules are structurally similar and the intermembrane helix parts are also similar to each other. The use of different tools in the software shows that the two molecules are structurally similar.

\subsection{Investigation of simulated results with COMSOL software}

In order to evaluate the simulation results obtained from COMSOL software, it was decided to transfer the drug into the vessel and finally to the vessels and capillaries that are found in large quantities in the liver. This study defined an input and an output for the vessel. Blood flow velocity input was determined to be vin $=0.008 \mathrm{~m} \mathrm{~s}^{-1}$ while the pressure output was determined to be $\mathrm{p}_{\mathrm{out}}=0$. The mole that penetrates the 
wall was clearly defined. The amount of drug penetration in the desired section was obtained. Nanoparticle motion was considered for different analyses and various factors such as field strength, blood flow velocity, particle density, etc., are effective factors in this simulation. Edward et al. ${ }^{21}$ investigated a model for the multifunctional magnetic particles of the vessels under contract. They concluded that the proposed model is embedded as a function of key variables including carrier particle size, properties and volume fraction of magnetic nanoparticles, and the magnetic properties showing the flow rate. Figure $2 \mathrm{~A}$ shows the concentration of the drug, the blue part being zero concentration and the red part showing the highest concentration. Figure $\mathbf{2 B}$ depicts the size of the velocity and the velocity field in the direction of the $Z$ axis. The velocity flow around the moving ring and the droplet moving through the edge of the old diffusion membrane is $0.25 \mathrm{~mm} \mathrm{~s}^{-1}$. Figure $2 \mathrm{C}$ illustrates the velocity of the fluid inlet and outlet flow lines and its representation. Figure 2D shows the drug concentration field inside the droplet as it passes through the edge of the permeable membrane. Line 0.8 is above the membrane.

Due to its blue color, the concentration is almost zero and the red color has the highest concentration, while the concentration is in the $0-12 \mathrm{~mol} \mathrm{~m}^{-3}$ range.

According to Figure 2D, the area attached to the membrane has the highest concentration. Figure $2 \mathrm{E}$ presents the last step and here the dissolved drug was examined at different times. The number obtained is 0.875 when a comparison was made with the research conducted by Chen et al. ${ }^{22}$, and it was the best and most appropriate time (Figure 2A). The movement of the connection point and contact with the wall where the defined value of the function is 1 and at the other points 0 was considered. The distance of our permeable membrane was assessed to ensure that the fluid inside the tube did not leak out (Figure 2B). The total dose of the drug in the drop is a function of time. We have plotted the total amount of moles in the drop, which is a drop speed of $0.1 \mathrm{~mm} \mathrm{~s}^{-1}$. Then the step velocity operator helped determine the step arrival distance from 0 to 1 . Figure $2 \mathrm{E}$ exhibits the different soluble concentrations from 0 to 10 , which is the time the drop starts to move to dissolve and finally stops.

The specified variable is defined as the distance from the highest drop point to the lowest $(0.24 \mathrm{~mm})$. When the solution is complete, the speed is reported at different times. The flow line shows the velocity from the drop inlet and outlet boundary. The total dose of the injected drug (drug concentration in the drop) was optimized for all velocities, which showed that as the droplet rate increased, the number of moles entering the droplet diminished. This occurred because the amount of drug that penetrates into the droplet depends on when the droplet comes into contact with the permeable membrane and the time taken to pass through it.

Figure 3 shows the drug dose injected into a drop as a function of time. As the droplet speed rises, the number of moles that enter the droplet begins to fall. This is because the amount of drug that penetrates into the droplet depends on when the droplet comes in contact with the permeable membrane. Figure $3 \mathrm{~A}$ shows the movement of the connection point and contact with the wall where the defined value of the function is 1 and at other points 0 is considered. The distance of the permeable membrane was checked to ensure that the fluid inside the tube did not leak. The specified distance of the obtained permeable 
membrane is the value of the function $6 \times 10^{-4} / 8 \times 10^{-4}$. The rest of the wall was 0 . Jie et al. ${ }^{23}$ investigated the biological activity and optimization of metallofullerene endohedral. They in fact reviewed the relevant data instead of the actual list and explained the nanocarrier as a diagnostic or therapeutic agent. Figure 3B shows that the total dose of the drug in the drop is plotted as a function of time. The total amount of moles inside the droplet is $0.1 \mathrm{~mm} \mathrm{~s}^{-1}$. The step velocity operator determines the step arrival distance from 0 to 1 . The scale is in time per second. The dose of the drug starts to increase over time (3 s) and remains constant after that (6s). Brazel and Pappas ${ }^{24}$ reported two types of polymers were employed to determine the validity of the model from the experimental results of drug release. The model is used to simulate experimental systems of moving boundaries to improve inflation behavior, and diffusion behavior in polymer networks.

Figure 4 is concerned with zero velocity. Figure $4 A$ shows the size of the starting surface velocity and Figure $4 \mathrm{~B}$ indicates the size of the final surface velocity. The solute transfer of the model devised for this study was selected. The input material was considered dilute. Particle transfer occurred only in the droplet section. Convection was adjusted according to the velocity flow. The system was adjusted to take the speed and concentration into account. The first velocity/time was set from 0 to 10 at which the solvent did not appear after the dissolution. The speed test is illustrated in Figure 4A, which is the last speed, and it is when our solution is completed that the best time and speed are identified $\left(U_{0}=0.001 \mathrm{~m} \mathrm{~s}^{-}\right.$ $\left.{ }^{1}\right)$. Figure $4 \mathrm{~B}$ is the first velocity that our solution has reached. The flow line is marked $\left(U_{0}=1.5 \times 10^{-4} \mathrm{~m} \mathrm{~s}^{-}\right.$ ${ }^{1}$ ). Skorb et al. ${ }^{25}$ explained that the combination of microscopic and spectroscopic methods served to analyze the structure and extent of optimization using ultrasound for the samples. The anti-corrosion activity of the new cerium / aluminum oxide system was demonstrated using a medical scanning method.

Figure $5 \mathrm{~A}$ shows the different moles and velocities from the time the drop started to the end, while Figure $5 \mathrm{~B}$ depicts the dose of drug injected into the drop as a function of time. The dose of the drug is vertical and the time is horizontal. Figure $5 \mathrm{~A}$ reveals that the total dose of injected drug (drug concentration in drops) was optimized according to all selected rates. Calculations showed that as the droplet velocity increased, the number of moles entering the droplet decreased. The amount of drug that penetrates into the droplet depends on when the droplet comes in contact with the permeable membrane and the time that passes through it. The speed diagram starts from the first time which is zero seconds and ends in about $6 \mathrm{~s}$. First, the droplet range shows the total molar amount of drug in the droplet. The last time is when the final concentration of the drug is completed. The graph is blue $\left(U_{0}=0.001 \mathrm{~m} \mathrm{~s}^{-1}\right)$. The first opportunity to commence the drug concentration was the water diagram $\left(U_{0}=1 \times 10^{-4} \mathrm{~m} \mathrm{~s}^{-1}\right)$. Figure $5 \mathrm{~B}$ presents the droplet speed increase and the number of moles that enter the droplet that decrease. The amount of drug that penetrates the droplet depends on when the droplet is directly related to: firstly, the permeable membrane; and secondly, how long the drug passes through it. Fundueanu et al. ${ }^{26}$ explained that the faster the drug penetrates into the drop, then time starts to run out. The molar amount of the drug also decreases. Polymers play an important role in determining the rate, persistence and penetration of 
nanoparticles in the body. Results of our computational studies show that success has been achieved in determining the rate at which nanoparticles penetrate the body.

\section{Conclusion}

First, according to VMD and COMSOL software, the desired molecules and proteins were examined and compared in terms of structural similarities and proper orientation. The results suggested the ideal position of the desired proteins and ligands. According to COMSOL software, relying on the magnetic properties and coating with polymers for slow release will inhibit enzyme activity and block the effects of opioids used to analyze drug delivery in the direction of toxin. The end resul is better detoxification in the liver. The best drug concentration detected is 1.5 seconds $U_{0}=2.510^{-4} \mathrm{~m} \mathrm{~s}^{-1}$ after entering the membrane of blood vessels. The best time of the dissolved drug according to the concentration study is $0.875 \mathrm{~s}$ at $\mathrm{U}_{0}=0.001 \mathrm{~m} \mathrm{~s}^{-1}$. This represents an innovation in the field of computational drug delivery. The results showed that iron nanoparticles were few and tolerable due to their toxicity, which is one of the best options for the body's organs. The main purpose of this study was to bring the drug to the desired point in order to improve people's ability to quit their addiction, better durability of the drug in the body and faster release of toxins by stimulating enzymes. We created the best possible conditions according to the pathways that include blood vessels and capillaries, so that the nanoparticles reached the vessels in the liver.

\section{Declarations}

Competing interests The authors declare no competing interests.

\section{Additional information}

Correspondence and requests for materials should be addressed to A.E.

\section{Author contributions}

S. M. and A.E. contributed to the all sections of the manuscript. All authors read and approved the final manuscript.

\section{Competing interests}

The authors declare no competing interests.

Ethics approval and consent to participate: Not applicable. We have not any animal experimental section in the text.

\section{References}


1. Huang, P., Kehner, G. B., Cowan, A. \& Liu-Chen, L.-Y. Comparison of pharmacological activities of buprenorphine and norbuprenorphine: norbuprenorphine is a potent opioid agonist. Journal of Pharmacology and Experimental Therapeutics 297, 688-695 (2001).

2. Kelly, K. L., Coronado, E., Zhao, L. L. \& Schatz, G. C. (ACS Publications, 2003).

3. Masoumi, S. \& Esmaeili, A. New method of creating hybrid of buprenorphine loaded rifampin/polyethylene glycol/alginate nanoparticles. International Journal of Biological Macromolecules (2020).

4. Hatorp, V., Hansen, K. T. \& Thomsen, M. S. Influence of drugs interacting with CYP3A4 on the pharmacokinetics, pharmacodynamics, and safety of the prandial glucose regulator repaglinide. The Journal of Clinical Pharmacology 43, 649-660 (2003).

5. Niemi, M., Neuvonen, P. J. \& Kivistö, K. T. The cytochrome P4503A4 inhibitor clarithromycin increases the plasma concentrations and effects of repaglinide. Clinical Pharmacology \& Therapeutics 70, 5865 (2001).

6. Zimmerman, W. B. (Beijing: China Communications Press, 2007).

7. Allen, B. \& Harocopos, A. Non-prescribed buprenorphine in New York City: motivations for use, practices of diversion, and experiences of stigma. Journal of substance abuse treatment $70,81-86$ (2016).

8. Draget, K., Bræk, G. S. \& Smidsrød, O. Alginic acid gels: the effect of alginate chemical composition and molecular weight. Carbohydrate Polymers 25, 31-38 (1994).

9. Heymann, J. B. \& Engel, A. Aquaporins: phylogeny, structure, and physiology of water channels. Physiology 14, 187-193 (1999).

10. Manikandan, P. \& Nagini, S. Cytochrome P450 structure, function and clinical significance: a review. Current drug targets 19, 38-54 (2018).

11. Mehmood, Z., Williamson, M. P., Kelly, D. E. \& Kelly, S. L. Metabolism of organochlorine pesticides: the role of human cytochrome P450 3A4. Chemosphere 33, 759-769 (1996).

12. Arnol'd, V. I. Mathematical methods of classical mechanics. Vol. 60 (Springer Science \& Business Media, 2013).

13. Humphrey, W., Dalke, A. \& Schulten, K. VMD: visual molecular dynamics. Journal of molecular graphics 14, 33-38 (1996).

14. Gear, C. W. Numerical initial value problems in ordinary differential equations. Prentice-Hall series in automatic computation (1971).

15. Quigley, D. \& Probert, M. Langevin dynamics in constant pressure extended systems. The Journal of chemical physics 120, 11432-11441 (2004).

16. Berjano, E. J. Theoretical modeling for radiofrequency ablation: state-of-the-art and challenges for the future. Biomedical engineering online 5, 1-17 (2006).

17. Bettaieb, A., Wrzal, P. K. \& Averill-Bates, D. A. Hyperthermia: Cancer treatment and beyond. Cancer treatment-conventional and innovative approaches, 257-283 (2013). 
18. Bhowmik, A., Repaka, R., Mishra, S. C. \& Mitra, K. Thermal assessment of ablation limit of subsurface tumor during focused ultrasound and laser heating. Journal of Thermal Science and Engineering Applications 8 (2016).

19. Samuels, E. R. \& Sevrioukova, I. F. An increase in side-group hydrophobicity largely improves the potency of ritonavir-like inhibitors of CYP3A4. Bioorganic \& medicinal chemistry 28, 115349 (2020).

20. Denker, B. M., Smith, B. L., Kuhajda, F. P. \& Agre, P. Identification, purification, and partial characterization of a novel $\mathrm{Mr} 28,000$ integral membrane protein from erythrocytes and renal tubules. Journal of Biological Chemistry 263, 15634-15642 (1988).

21. Furlani, E. J. \& Furlani, E. P. A model for predicting magnetic targeting of multifunctional particles in the microvasculature. Journal of Magnetism and Magnetic Materials 312, 187-193 (2007).

22. Chen, W. et al. Novel coronavirus international public health emergency: guidance on radiation oncology facility operation. Advances in Radiation Oncology (2020).

23. Meng, J. et al. Biomedical activities of endohedral metallofullerene optimized for nanopharmaceutics. Journal of nanoscience and nanotechnology 10, 8610-8616 (2010).

24. Brazel, C. S. \& Peppas, N. A. Modeling of drug release from swellable polymers. European journal of pharmaceutics and biopharmaceutics 49, 47-58 (2000).

25. Skorb, E., Shchukin, D., Möhwald, H. \& Andreeva, D. Sonochemical design of cerium-rich anticorrosion nanonetwork on metal surface. Langmuir 26, 16973-16979 (2010).

26. Fundueanu, G., Constantin, M. \& Ascenzi, P. Preparation and characterization of pH-and temperaturesensitive pullulan microspheres for controlled release of drugs. Biomaterials 29, 2767-2775 (2008).

\section{Scheme}

Scheme 1 is available in supplementary section.

\section{Figures}




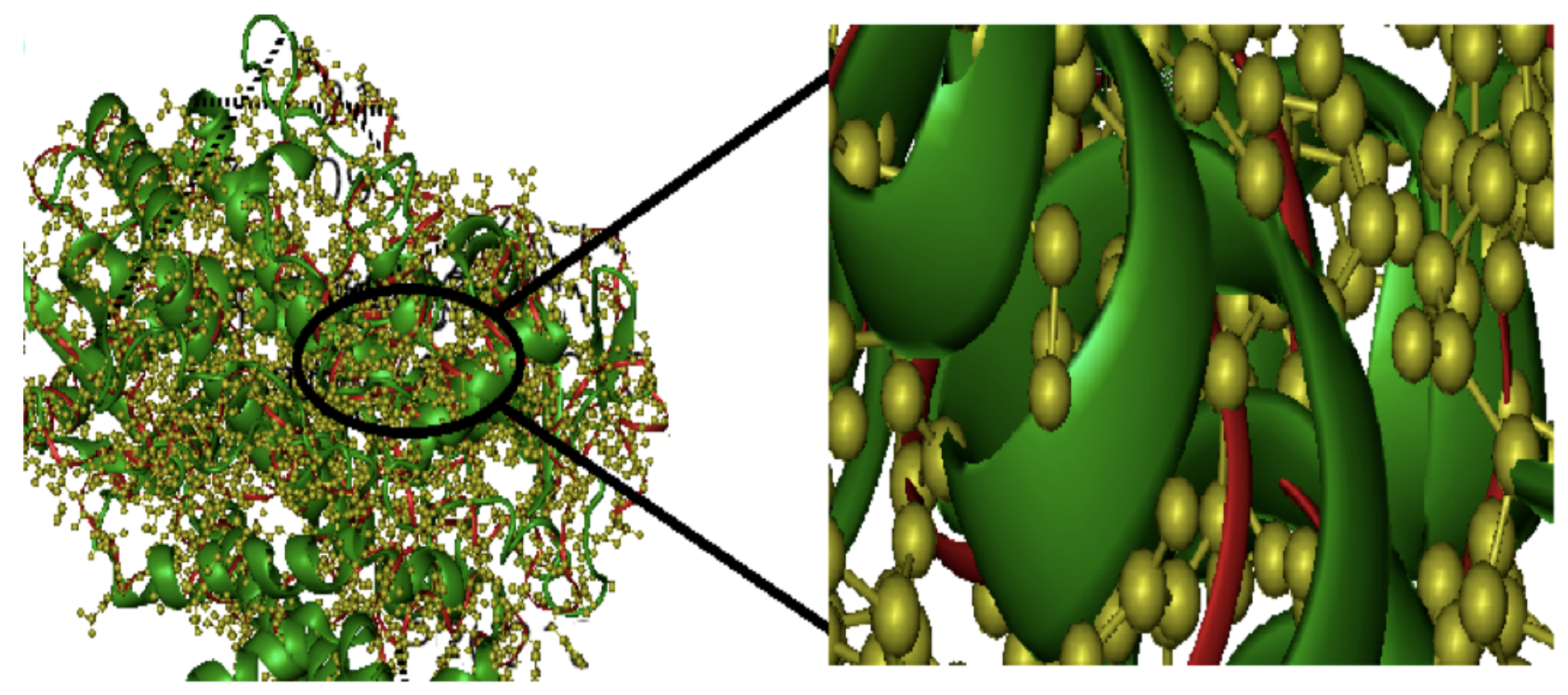

Figure 1

Demonstration of stained molecules of amino acids and proteins with structural similarities after examination of a specific site. 

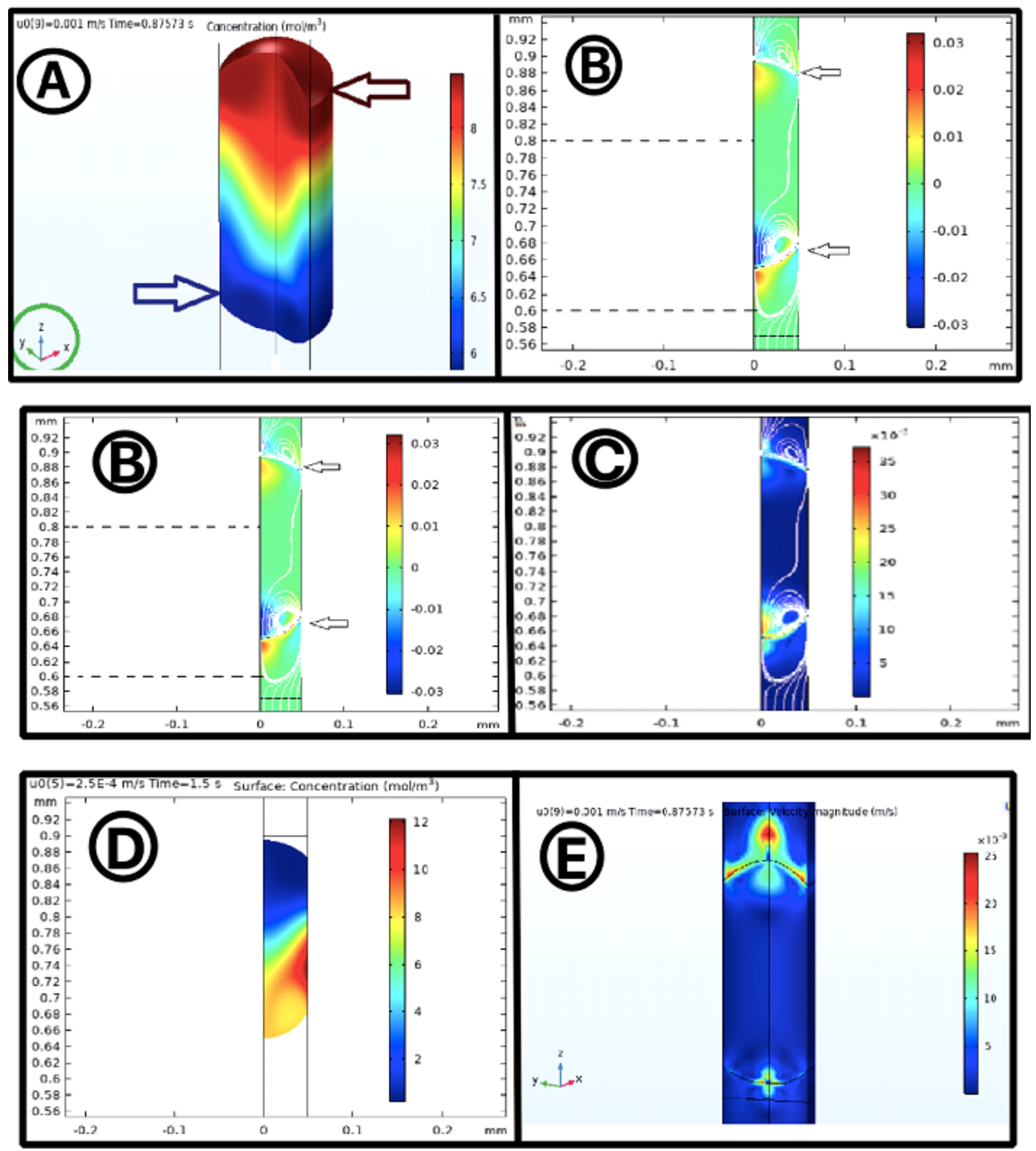

Figure 2

(A) Displays the concentration of drug within the drop; (B) Displays the field and speed size; (C) Input and output flow line; (D) Concentration of the drug in drops from the edge of the membrane (E) Drug dissolved at different times. 

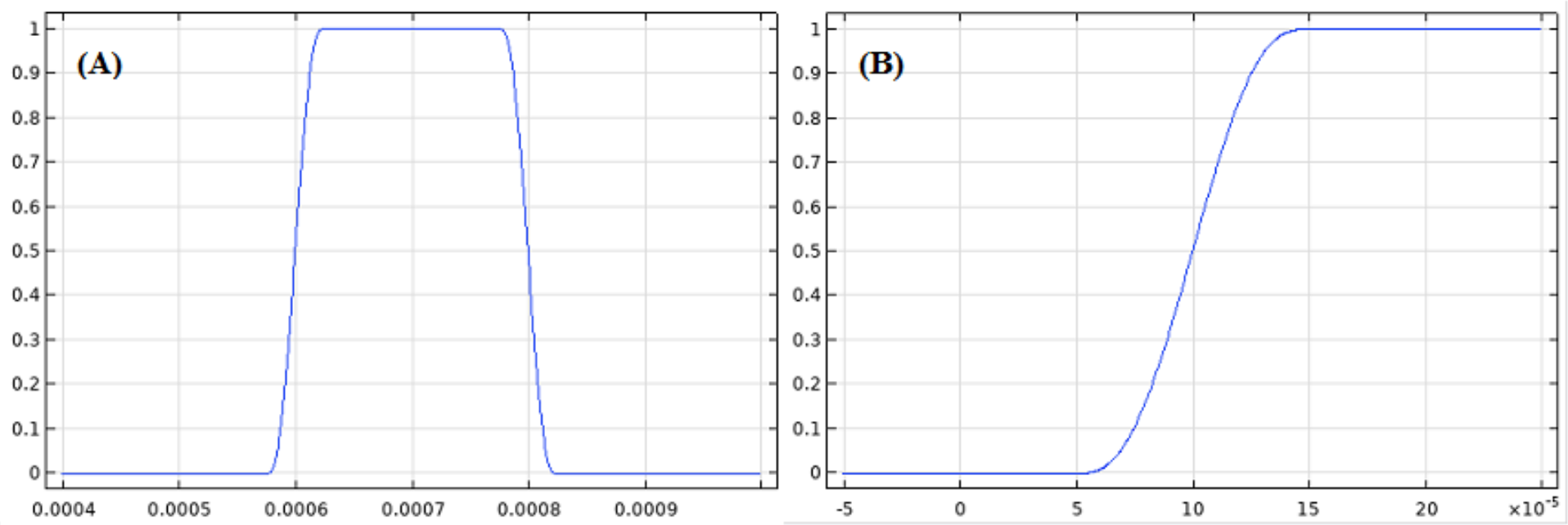

Figure 3

(A) Investigating the movement of the fluid connection from the beginning to the end; (B) Total drug doses in drops over time.

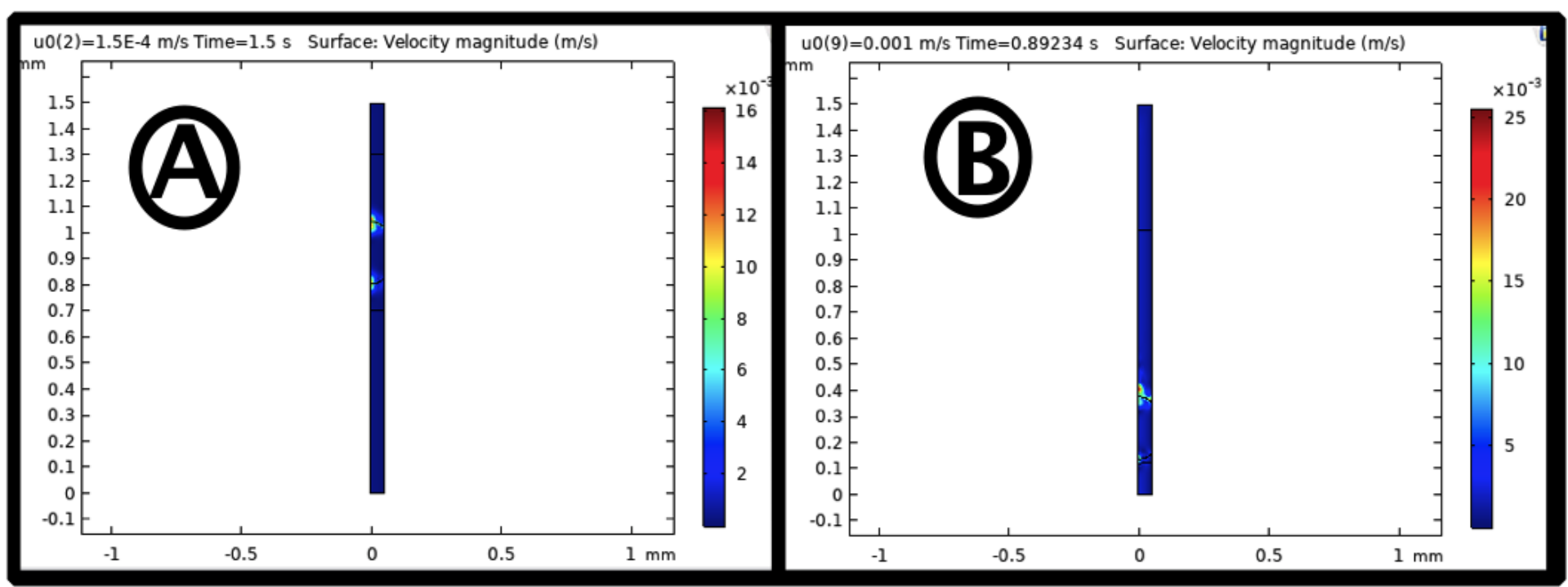

Figure 4

Investigating different concentrations within drops of time (A) Checking the size of the starting surface velocity; (B) Checking the size of the final surface velocity. The graph shows the total molar amount of the drug inside the droplet. Our $\mathrm{x}$-axis is marked. with a function of time. 

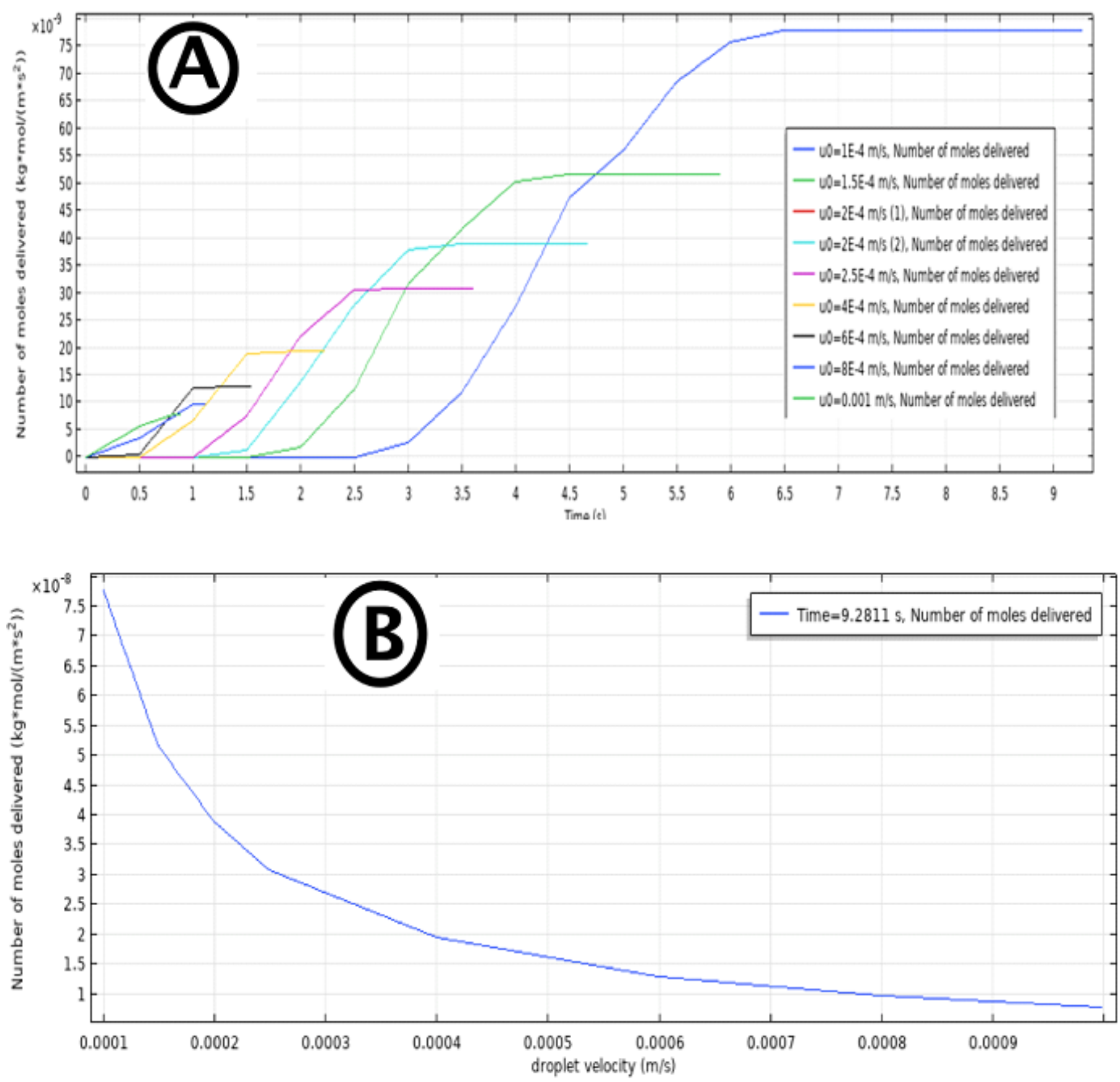

Figure 5

(A) Investigating the different moles and velocities from the time the drop starts to the end; (B) The total dose of drug injected into the drop as a function of time.

\section{Supplementary Files}

This is a list of supplementary files associated with this preprint. Click to download. 
- floatimage1.png

Page 15/15 